\title{
THE ORNL FAST-WAVE ICRF ANTENNA FOR ALCATOR C-MOD*
}

\author{
R. H. Goulding, D. J. Hoffman, D. L. Conner, C. J. Hammonds, J. L. Ping, B. W. Riemer, \\ P. M. Ryan, D. J. Taylor, R. B. Wysor, and J. J. Yugo ${ }^{\dagger}$ \\ Oak Ridge National Laboratory, P.O. Box 2009, Oak Ridge, Tennessee 37831-8071
}

\section{ABSTRACT}

A fast-wave ICRF antenna is being designed for Alcator C-Mod which is prototypical in many respects of the baseline launcher design for the Compact Ignition Tokamak (CIT). The C-Mod launcher has a single current strap, with a strap and cavity geometry very similar to one quadrant of the CIT launcher, which has four straps in a 2 by 2 configuration. The antenna fits entirely within an 8 -in.-wide by 25 -in.-long port and is radially movable over a distance of $15 \mathrm{~cm}$. It will operate at a frequency of $80 \mathrm{MHz}$ for pulse lengths up to $1 \mathrm{~s}$, at a maximum power level of $2 \mathrm{MW}$, corresponding to a power flux of $>1.5 \mathrm{~kW} / \mathrm{cm}^{2}$. The antenna is an end-fed, double-loop configuration in which the current strap is grounded in the middle to provide mechanical support. The design includes a disruption support system that accommodates thermal expansion of the antenna box while supporting large disruption loads. It also includes a novel matching system consisting of an external resonant loop with two shunt capacitors serving as tuning/matching elements.

\section{INTRODUCTION}

A high-power-density, fast-wave, ion cyclotron range of frequencies (ICRF) antenna is being designed at Oak Ridge National Laboratory (ORNL), which will incorporate many features relevant to operation in nextgeneration tokamak experiments. The antenna will be installed and operated in the Alcator C-Mod Tokamak. It is specifically designed to be prototypical, in as many aspects as possible, of the launcher currently proposed for use on CIT. ${ }^{1}$ The antenna will operate at power levels up to $2 \mathrm{MW}$, for pulse lengths up to $1 \mathrm{~s}$, at a frequency of $80 \mathrm{MHz}$, which corresponds to $\Omega_{3_{\mathrm{He}}}$ at 7.9 $\mathrm{T}$. The antenna is a single-strap, end-fed configuration with the strap grounded at the center.

Several notable features of the design make it well suited to application in next-generation tokamaks. It features a disruption support system able to withstand loads generated by a disruption of $3 \mathrm{MA} / 3 \mathrm{~ms}$ at $9 \mathrm{~T}$ while allowing thermal expansion of the antenna box relative to the port in which it is located or, conversely, contraction of the port. The design of the disruption support structure allows installation or removal of the entire launcher, including supports from outside the port, greatly simplifying remote maintenance.

The Faraday shield elements, which are cylindrical Inconel rods, are designed to have very low primary thermal stresses, facilitating the handling of high power densities. In the CIT Faraday shield, these solid Inconel rods can be replaced by tubes, allowing direct liquid cooling of shield elements without changing the electrical characteristics of the shield design.

Ceramic feedthroughs are located outside the vacuum vessel, where exposure to ionizing radiation, which could enhance rf losses and seriously degrade the feedthrough life expectancy, ${ }^{2}$ can be limited. There are no ceramic supports between the current strap and these feedthroughs.

The antenna is tuned and matched by using an external resonant loop employing integral capacitors to achieve a match at the loop feed point. This system is capable of meeting the $2-\mathrm{MW} / \mathrm{strap}$ power requirement while being compact enough to allow the entire matching system to fit within the CIT shield wall, eliminating spurious resonances and power-handling difficulties caused by long unmatched transmission line runs. It also allows tuning in two to three frequency bands without modifying the physical layout of the system. ${ }^{1}$

\section{MECHANICAL DESIGN}

\section{General Description}

Figure 1 is a side view of the launcher. The current strap, antenna backplane, antenna cavity, and Faraday shield components are all fabricated from Inconel to withstand forces generated by disruptions. Molybdenum bumpers are provided to protect the sidewalls of the antenna. Current-carrying surfaces are all plated with copper or silver. The current strap is $8 \mathrm{~cm}$ wide and $48 \mathrm{~cm}$ in total length. It is grounded at the center through a support that is bolted to the antenna backplane. The minimum distance from the back of the current strap to the backplane is $8.8 \mathrm{~cm}$. The antenna cavity is $13.3 \mathrm{~cm}$ wide and has solid sidewalls extending

*Research sponsored by the Office of Fusion Energy, U.S. Department of Energy, under contract DE-AC05-84OR21400 with Martin Marietta Energy Systems, Inc.

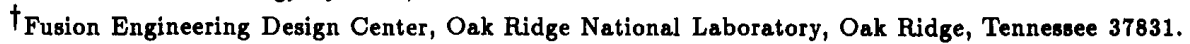




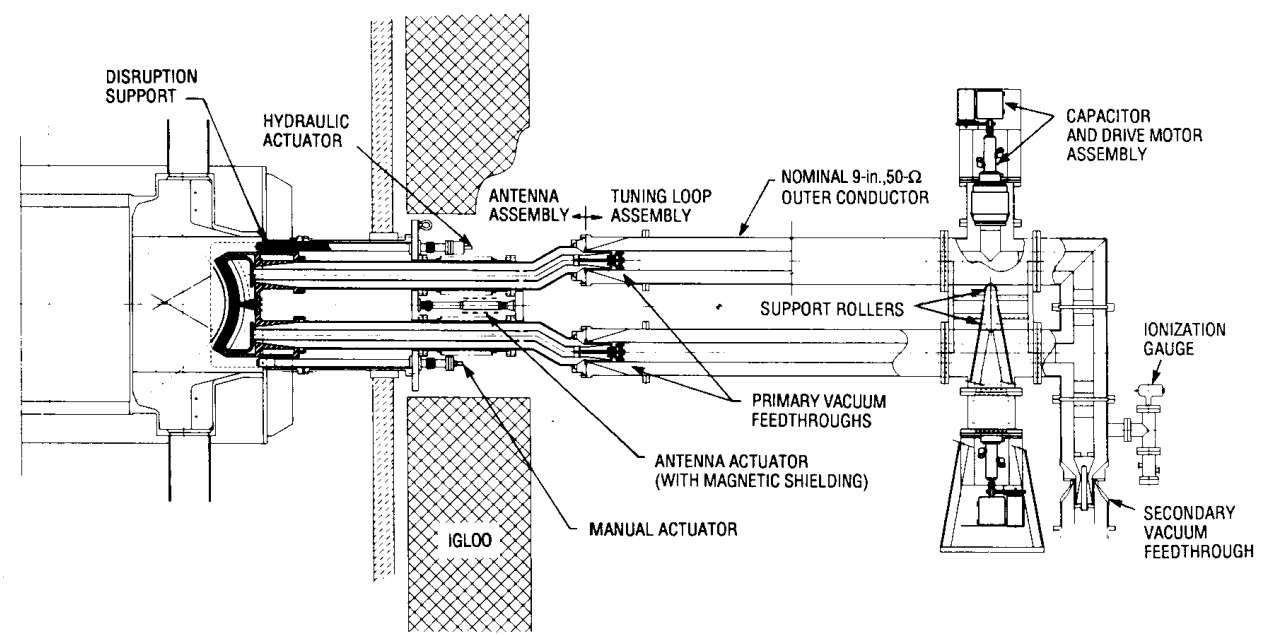

Fig 1. The ORNL antenna for Alcator C-Mod.

from the backplane to within $1 \mathrm{~cm}$ of the back of the current strap.

There are a total of 56 Faraday shield elements consisting of cylindrical Inconel rods bent into a flattened U shape (Fig. 2.) and arranged in two tiers. The front rods are $1.27 \mathrm{~cm}$ in diameter, and the rear rods are 0.95 $\mathrm{cm}$ in diameter. The rods in the front tier are ground flat at the front, reducing the total shield thickness. All rods are plated with copper and then coated with a layer of titanium carbonitride $\sim 7 \mu \mathrm{m}$ thick. The rods

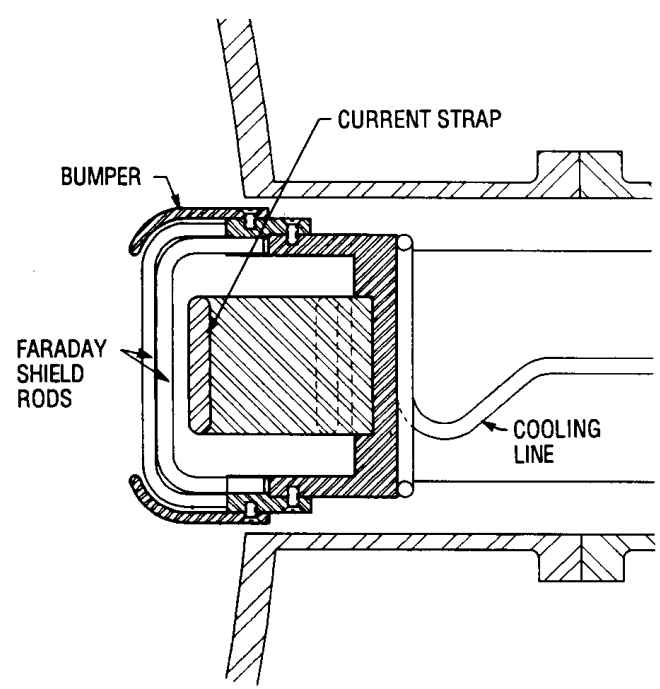

Fig 2. Top view showing Faraday shield tubes. are welded into a support frame that is bolted to the antenna box sidewalls.

The antenna is supported at the front by the disruption support system, described later in more detail. Additional support is provided by rollers attached to flanges in the resonant loop coaxial line, as shown in Fig. 1. Radial loads resulting from disruptions and vacuum loads are carried by a support yoke that is connected by a motor-driven actuator to the port cover plate, allowing radial translation of the antenna.

Power is fed to the ends of the straps by two coaxial, $50-\Omega$, copper-plated stainless steel transmission lines. The outer conductors have an outer diameter of $140 \mathrm{~mm}$ and a wall thickness of $6 \mathrm{~mm}$. Bellows are located in the center conductor lines between the current strap and the vacuum feedthroughs to allow thermal expansion of the center conductors relative to the outer conductor and to reduce transmission of disruption loads from the current strap to the ceramic feedthroughs. The primary vacuum feedthroughs for the antenna are located $1.7 \mathrm{~m}$ from the current strap ends. The design of these feedthroughs has been described elsewhere. ${ }^{3}$ The balance of the resonant circuit beyond the feedthroughs is fabricated from standard $230-\mathrm{mm}, 50-\Omega$ copper transmission line. A secondary vacuum feedthrough beyond the resonant loop feed point (Fig. 1) allows this section of line to be operated under high $\left(10^{-7}\right.$ Torr) vacuum, separately from the machine vacuum. This is done to allow operation with a high voltage standing wave ratio (VSWR) without requiring pressurization with $\mathrm{SF}_{\theta}$, which is not acceptable for use on CIT. A 5-cm pumpout port is provided between this feedthrough and the tee. The flanges on the transmission line will be modified if necessary to achieve the required vacuum. 


\section{Disruption Support Mechanism}

The disruption support mechanism is designed to allow the entire antenna, including disruption supports, to be installed from outside the vacuum vessel, considerably simplifying remote handling in a CIT type of environment. It is designed to carry transverse loads only, which are predicted to be much larger than radial loads, according to force calculations performed for a similar ICRF antenna designed by the Massachusetts Institute of Technology for use in Alcator C-Mod. ${ }^{4}$ Elimination of gaps between contacting surfaces allows large loads to be carried, but the design also allows for thermal expansion of the antenna box relative to the port in which it is supported.

The present design for the support mechanism (Fig. 3) calls for two identical assemblies located at the top and bottom of the antenna box. Each assembly includes a semicylindrical wedge swivel (or key), which fits into a wedge way running along the top or bottom of the antenna box, and a bearing/wedge housing, which supports bearing pads that contact the vacuum vessel port wall at the joint between the vacuum vessel and the port nozzle. Loads are transmitted to the housing through two adjustable wedges in the form of truncated hexagonal pyramids. The shape was chosen to allow surface contact as the wedges are moved in and out. The pyramids are tapered at an angle of $8^{\circ}$, which is 2.5 to 4 times the self-locking angle, so that the wedges will move apart in response to thermal contraction of the port (or expansion of the antenna frame).

The wedges in the bottom assembly are adjusted by means of a nut, which in the C-Mod design cannot be adjusted remotely. The force on the top wedges is applied through a 5-cm-ID hydraulic cylinder, with the outer wedge connected to the piston by a rod and the inner wedge connected to the cylinder by a concentric sleeve. Pressure can be applied to either side of the piston, serving either to force the wedges toward each other and preload the bearing pads during normal operation or to break the wedges free should it become necessary in order to permit radial movement of the antenna. The key and beam are held in a fixed position relative to the port by a cylindrical support extending back to the port cover plate.

All sliding surfaces are coated with a $1-\mu$ m-thick layer of tungsten disulfide that is diffusion-bonded to the metal surfaces. This coating serves as a lubricant and prevents vacuum welding. It is particularly well suited for this application in that it has a low friction

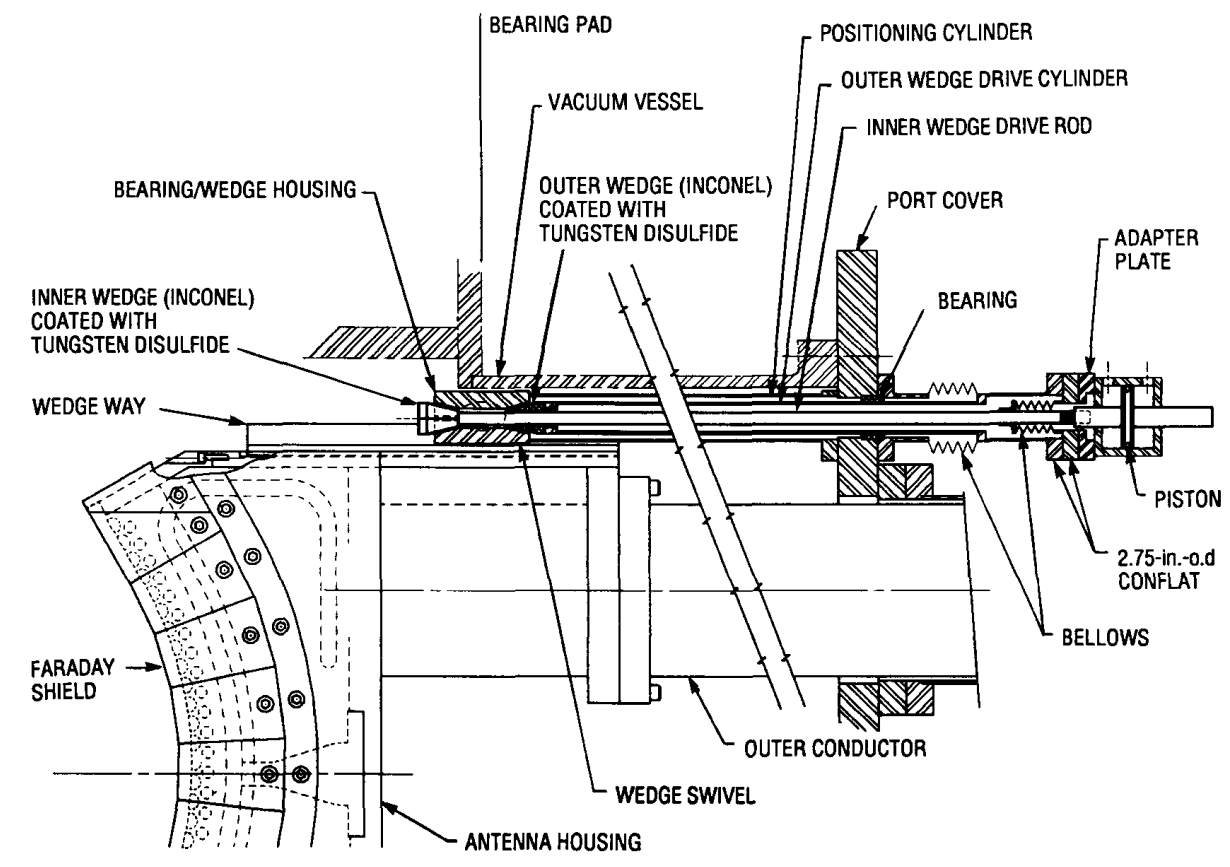

Fig 3. Disruption support mechanism. 
coefficient (0.025-0.065), a high load capacity, a wide range of operating temperatures, and a very low vapor pressure after bonding.

During operation, a constant force is applied to the piston; thus, the support mechanism self-adjusts for relative thermal expansion of the antenna frame. Since the response time of the system is slow compared with the time scale of a disruption, in this case the full loads are transmitted through the wedges to the vacuum vessel.

\section{Antenna Cooling}

An extensive analysis of antenna heating due to both $\mathrm{rf}$ and plasma sources is currently under way. Preliminary results indicate that Faraday shield tubes can be held to safe temperatures $\left(\sim 600^{\circ} \mathrm{C}\right.$ maximum) for the specified pulse duration ( $1 \mathrm{~s}$ rf, $3 \mathrm{~s}$ plasma every $20 \mathrm{~min}$ ) if the backplane is maintained at a fixed $30^{\circ} \mathrm{C}$ temperature by active cooling. A system for trace cooling of the back side of the backplane has been incorporated into the design, but the cooling medium has not yet been decided upon. Further work is currently under way to better model the power deposition profiles on the Faraday shield and to determine the cooling requirements necessary to achieve a considerably higher rf duty cycle during antenna conditioning.

\section{ELECTRICAL DESIGN}

\section{Antenna/Cavity}

The current strap is an end-fed, double-loop design that is grounded at the center. We have examined means of optimizing the current strap, Faraday shield, and cavity geometry in order to maximize the powerhandling capability of the antenna.

Power Handling Optimization. One optimization parameter that has been examined is the ratio of the peak rf power dissipation to the value averaged over the region in which the power dissipation is nonnegligible. This parameter determines in part the peak temperature reached on the shield after a pulse. This quantity was determined for a range of current strap widths by calculating the rf magnetic field amplitudes at the surface of the shield elements (without the elements present) with a two-dimensional (2-D) magnetostatic code ${ }^{5}$ that includes the effects of the current strap and cavity geometry. The effect of the shield elements on the field is primarily to increase the amplitude of the field components normal to the axis of the rods in the gaps between them. We included this effect by using results of an analogous 2-D electrostatic calculation, as described in ref. 6 . Results of the calculation have shown that varying the current strap width has little effect on the ratio of peak-to-average power dissi- pation levels (as defined previously) and serves only to change the locations of the peak dissipation regions.

Other optimization strategies considered were the minimization of the peak voltages on the current strap and the tuning loop for a given value of coupled power. The peak voltage on the current strap for a given power level is proportional to $L / R^{1 / 2}$, where $L$ is the inductance per unit length of the strap and $R$ is the resistance (including radiation resistance) per unit length. The peak voltage in the resonant loop is proportional to the square root of the VSWR in the lines between the current strap and the tuning capacitors.

Transmission Line Analysis. The 2-D magnetostatic code referenced previously was used to determine transmission line parameters for the current strap for use in a transmission line analysis. Values of $\mathrm{L}$ and relative values of $\mathrm{R}$ for different strap widths and different slot lengths in the sidewalls were obtained. The absolute magnitude of $R$ depends on the plasma surface impedance, which is not modeled in the code. The code treats the plasma as a perfectly conducting surface. Relative changes in $R$ due to changes in antenna geometry are accurately modeled by the code, as was demonstrated in low-power loading measurements performed on the Doublet III-D tokamak. ${ }^{7}$ The strap capacitance per unit length is not calculated in the code but was assumed to remain constant as the slot length is changed. This is a reasonable assumption since the capacitance is expected to change very little if the slotted sidewall is replaced by a continuous wall. On the other hand, as the strap width is varied, the capacitance changes with the change in surface area. In this case, it was assumed that the phase velocity remains constant.

With the preceding assumptions and an arbitrary value of 0.54 for the phase velocity for the case in which the side slots extend $1 \mathrm{~cm}$ behind the current strap (based on measurements from a different antenna), a transmission line analysis was used to determine the maximum strap and resonant loop voltages as a function of strap width and slot length. The absolute magnitude of the resistive loading was taken to be a conservative value of $8 \Omega / \mathrm{m}$ for the case of an 8 -cm-wide strap with side slots extending $1 \mathrm{~cm}$ behind the strap. The results [Figs. 4(a) and 4(b)] show that increases in strap width have a relatively small effect on the magnitude of the peak tuning loop voltage but that increasing the slot length has a larger effect, caused by the effect on $R$, to which the VSWR is relatively sensitive. On the other hand, increasing the strap width does significantly decrease the maximum strap voltage, whereas increasing the slot length has relatively little effect. Since minimizing the voltage in both regions is desirable, the antenna can be optimized with respect to both parameters by using a wide current strap and making the side 

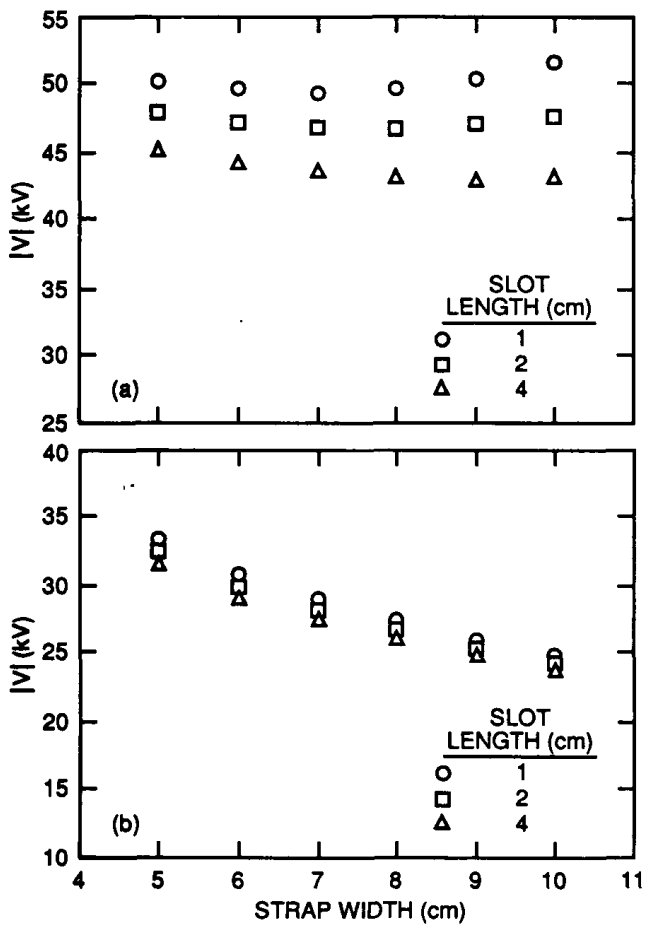

Fig 4. (a) Effect of changes in current strap width and distance that slot extends behind strap on peak resonant loop voltage. (b) Effect of these changes on peak voltage on the current strap.

slots as long as possible. In the C-Mod design the 8cm strap width was chosen to allow a $2.5-\mathrm{cm}$ toroidal gap between the current strap and the Faraday shield tubes, which are not magnetically insulated from one another. The $1-\mathrm{cm}$ extent of the slots behind the current strap was chosen for mechanical reasons, but the possibility of extending them further is being examined. It is important to point out, however, that even without further optimization, the calculated maximum voltages of $28 \mathrm{kV}$ on the current strap and $50 \mathrm{kV}$ in the resonant loop for $2 \mathrm{MW}$ of input power are within an acceptable range and have been handled successfully on ICRF antennas operated on the Tokamak Fusion Test Reactor. ${ }^{8}$ A mockup following the actual antenna geometry will be built in the near future to obtain more accurate values of the current strap transmission line parameters.

\section{Tuning/Matching System}

A simplified schematic of the antenna circuit is shown in Fig. 5. The two ends of the current strap are fed by an external section of transmission line to form

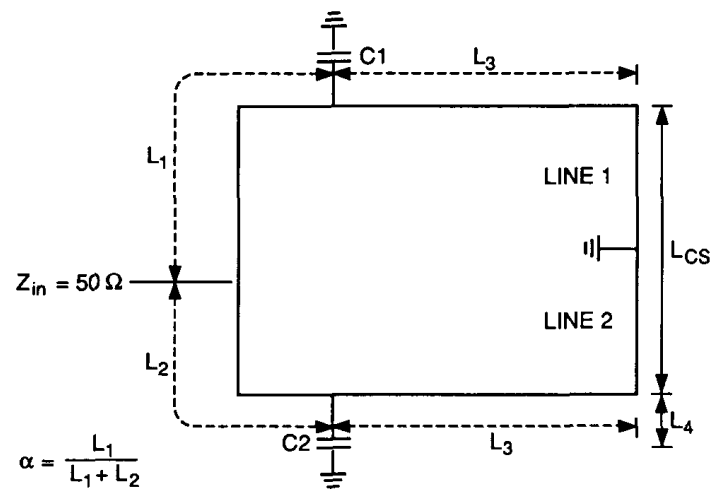

Fig 5. Schematic of antenna/tuning loop circuit.

a loop. Two short shunt lines are terminated in capacitances and located near the feed point of the loop. The capacitors to be used in the actual circuit are Comet CV7W units having a capacitance range of $15-150 \mathrm{pF}$, a peak working voltage of $55 \mathrm{kV}$, and a current limit of $\sim 850 \mathrm{~A} \mathrm{rms}$ in continuous operation at $80 \mathrm{MHz}$.

The capacitances required for a match at the feed point can be determined as follows. With a simple impedance transformation and the assumption of ideal lines (except for the current strap), the susceptances looking into both legs at the tee are given by the expression

$$
B_{i T}=-\frac{1}{Z_{0} \tan \left(\beta l_{i}\right)} \pm \sqrt{\frac{G_{i T} / Z_{0}^{2}}{G_{i C} \sin ^{2}\left(\beta l_{i}\right.}}-G_{i T}^{2},
$$

where $B_{i T}$ is the susceptance looking into leg $i$ at the tee, $G_{i C}$ is the conductance at the capacitor in line $i, G_{i T}$ is the value of the conductance of line $i$ at the tee, $Z_{0}$ is the characteristic impedance of the lines between the capacitors and the tee, and $l_{i}$ is the length of line $i$ between the tee and capacitor $i$. The value of $G_{i C}$ can be calculated from the strap input impedance if the length of the line between the strap and capacitor is known; thus, if all transmission line lengths are specified, all of the quantities on the right-hand side of the equation are known except for $G_{i T}$. The negative root gives the value for the longer line, and the positive root gives the value for the shorter line.

To match to the feed line at the input to the tee, we require that $B_{2 T}=-B_{1 T}$ and that $G_{1 T}+$ $G_{2 T}=1 / Z_{\text {in }}$, where $Z_{\text {in }}$ is the feed line characteristic impedance. Using these requirements and Eq. (1), we can obtain the values of $G_{1 T}, G_{2 T}, B_{1 T}$, and $B_{2 T}$. Alternatively, we can add the requirement that $G_{2 T}=G_{1 T}$, which is necessary if the amplitudes of the currents on the two halves of the strap are to be exactly 
equal at any given distance from the short. In this case, if $l_{1}$ is given, we can use Eq. (1) to calculate $l_{2}$, which tells us the optimum tap point. Once these values have been determined in the manner outlined previously, the values $B_{i C}$ of the susceptances on the generator side of the capacitors can easily be determined. The values of the susceptances on the load side can also be determined, and the difference between the two values can be used to determine the capacitances required to obtain a match.

The procedure outlined here has been used to determine matching capacitances for a range of plasma loading conditions. Results shown in Fig. 6 indicate that a wide range of resistive and reactive loads can be matched with small variations in the matching capacitances. The parameters used in these calculations are given in Table I.

Table I. Transmission line parameters for capacitance calculations

\begin{tabular}{lllll}
\hline Line & $R(\Omega / \mathrm{m})$ & $Z_{0}(\Omega)$ & $v / c$ & Length $(\mathrm{m})$ \\
\hline $\begin{array}{l}\text { Current } \\
\text { strap }\end{array}$ & $6-20$ & 32 & 0.54 & 0.48 \\
1 & 0 & 50 & 1.0 & 1.2 \\
2 & 0 & 50 & 1.0 & 0.6 \\
3 & 0 & 50 & 1.0 & 3.71 \\
4 & 0 & 50 & 1.0 & 0.08 \\
\hline
\end{tabular}
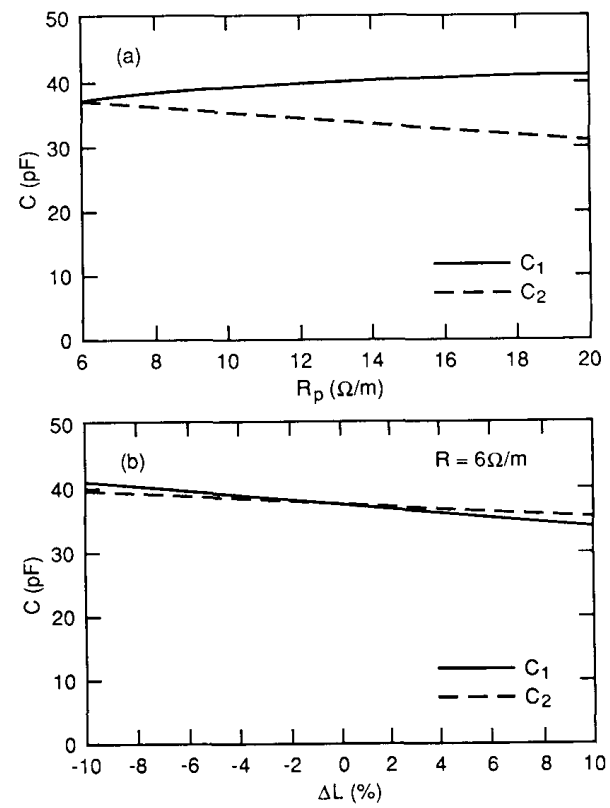

Fig 6. (a) Variation of matching capacitances with changes in resistive loading. (b) Variation of matching capacitances with changes in reactive loading.

\section{CONCLUSIONS}

The fast-wave ICRF launcher designed by ORNL for use on Alcator C-Mod incorporates many features based on the requirements of next-generation large tokamak experiments. One of these features is a disruption support mechanism that can handle large loads while allowing thermal expansion of the antenna frame relative to the port as well as installation and removal of the antenna from outside the port. Another feature is a low-thermal-stress, high-transparency Faraday shield design that can be adapted to active cooling. A third feature is the minimization of the use of ceramic supports in the antenna transmission line and the location of ceramic vacuum feedthroughs as far from the plasma as possible. A fourth is the use of an external tuning loop with integral tuning elements, allowing tuning in two to three frequency bands and matching to a range of plasma loads at the loop feed point, which can be located well within the location of the shield wall. Considerable effort is being applied to optimize the design to allow operation at power levels up to $2 \mathrm{MW}$.

\section{REFERENCES}

[1] R. H. Goulding et al., "ICRF Antenna Designs for CIT and Alcator C-Mod," AIP Conf. Proc., vol. 190, pp. 250-253, 1989.

[2] S. N. Buckley and P. Agnew, "Radiation-Induced Changes in the Dielectric Properties of Insulating Ceramics at ICRH Frequencies," J. Nucl. Mater., vol. 155-157, pp. 361-365, 1988.

[3] T. L. Owens, F. W. Baity, and D. J. Hoffman, "ICRF Antenna and Feedthrough Development at the Oak Ridge National Laboratory," AIP Conf. Proc., vol. 129, pp. 95-98, 1985.

[4] Y. Takase et al., "Alcator C-Mod ICRF Fast Wave Antenna Design and Analysis and Expected Performance," AIP Conf. Proc., vol. 190, pp. 346-349, 1989.

[5] P. M. Ryan et al., in Proceedings of the 15th European Conference on Controlled Fusion and Plasma Heating, Dubrovnik, Yugoslavia, May 16-20, 1988. European Physical Society, 1988, part II, p. 795.

[6] P. M. Ryan et al., paper presented at the IAEA Technical Committee Meeting on Ion Cyclotron Resonance Heating/Edge Physics, Garching, FRG, October 2-5, 1989.

[7] P. M. Ryan et al., "Magnetostatic Analysis of ICRH Antenna Structures: Application to the DIII-D RF Coupling Improvements," Bull. Am. Phys. Soc., vol. 33, p. 2017, 1988.

[8] D. J. Hoffman, Oak Ridge National Laboratory, private communication, 1989. 